\title{
MODELLING OF BIOLOGICAL TISSUE DAMAGE PROCESS WITH APPLICATION OF INTERVAL ARITHMETIC
}

\author{
Anna Korczak, MareK Jasiński \\ Silesian University of Technology, Institute of Computational Mechanics and Engineering, Gliwice, Poland \\ e-mail: anna.korczak@polsl.pl; marek.jasinski@polsl.pl
}

In the paper, the numerical analysis of thermal processes proceeding in a $2 \mathrm{D}$ soft biological tissue subjected to laser irradiation is presented. The transient heat transfer is described by the bioheat transfer equation in Pennes formulation. The internal heat source resulting from the laser-tissue interaction based on the solution of the diffusion equation is taken into account. Thermophysical and optical parameters of the tissue are assumed as directed intervals numbers. At the stage of numerical realization. the interval finite difference method has been applied. In the final part of the paper, the results obtained are shown.

Keywords: directed interval arithmetic, bioheat transfer, optical diffusion equation, Arrhenius scheme

\section{Introduction}

One of the common problems in mathematical modelling of biological systems is the significant variation in the parameters that may result from, among others, individual characteristics. For some parameters, imprecisions in the estimation of their values may be a result of different measurement methods or specific features of tissues. The measurement is not often possible in a living organism. This may cause the values of individual parameters found in the literature to differ significantly from one another. It is also worth noting that tissue parameters may have different values depending on their degree of thermal damage. For example, the value of the scattering coefficient of tissue during the tissue damage process increases several times, what could be observed as "whitening" of the tissue (Jasiński, 2015).

One of the most widely used mathematical tools to take into account uncertainties of parameters are sensitivity analysis methods. By using them, very different problems were analysed, also in the field of heat transfer in the living organism (Jasiński, 2014; Kałuża et al., 2017; Mochnacki and Ciesielski, 2016). The approach using interval or fuzzy numbers is slightly less frequently used, although one can find works in which thermal processes in the human skin or eye are under consideration (Jankowska and Sypniewska-Kaminska, 2012; Mochnacki and Piasecka-Belkhayat, 2013; Piasecka-Belkhayat and Jasiński, 2011). The other initial-boundary value problems for partial differential equations were also considered in many fields of science (Gajda et al., 2000; Di Lizia et al., 2014; Nakao, 2017).

One can distinguish between two types of interval arithmetic: directed and classical (Dawood, 2011; Hansen and Walster, 2004; Markov, 1995; Popova, 1994). Classical interval arithmetic was proposed in the 60s of the last century by Moore (1966). The two kinds of arithmetic differ mainly in the definition of an interval and the set of arithmetic operations. A classical interval $\bar{x}=[a, b]$ is defined as a set of real numbers that are between the lower bound $a$ and upper bound $b$ like $\bar{x}=\{x \in \mathbb{R} \mid a \leqslant x \leqslant b\}$ while a directed interval is defined by only a pair of real numbers as $\bar{x}=[a, b]$ where $a, b \in \mathbb{R}$. Because of that, during successive calculations based on the classical interval arithmetic and many computational methods, including methods based 
on finite differences applied, the widths of intervals increase what leads to the so-called range overestimation problem (Hansen and Walster, 2004; Markov, 1995; Nakao, 2017). Using the directed interval arithmetic, it is possible to obtain the point (degenerated) interval $[0,0]=0$ by subtraction of two identical intervals $\bar{a}-\bar{a}=[0,0]$ and the point interval $[1,1]=1$ as the result of the division $\bar{a} / \bar{a}=1$, which is impossible when applying the classical interval arithmetic. From a practical point of view, it is the most important one and causes the directed interval arithmetic to be popular in numerical applications (Dawood, 2011; Piasecka-Belkhayat and Korczak, 2016, 2017).

Modelling of laser-biological tissue interactions requires appropriate mathematical description. It is known that the scattering dominates over the absorption in soft tissues for wavelengths between 650 and 1,300 nm (so-called biological window). Because of this, usually the radiative transport equation is taken into account (Dombrovsky and Baillis, 2010). There are several modifications in the discrete ordinates method or statistical Monte Carlo methods which are used to solve this equation (Banerjee and Sharma, 2010; Welch, 2011). In some cases, it is possible to approximate the light transport using the diffusion equation (Dombrovsky et al., 2013; Fasano et al., 2010; Jacques and Pogue, 2008).

Modelling of the laser energy deposition is the first step in the modelling of physical processes proceeding in biological tissues subjected to a laser beam. Next, the temperature distribution must be calculated by making use of the bioheat transfer equation. The Pennes equation is the earliest one known but is probably still the most popular and widely used (Abraham and Sparrow, 2007; Majchrzak and Mochnacki, 2017; Paruch, 2014). The newest achievements in this field are based on the porous media theory (GDPL equation, generalized dual-phase lag equation) which takes into account the heterogeneous structure of biological tissue (Jasiński et al., 2016; Majchrzak and Mochnacki, 2017; Majchrzak et al., 2015).

The last step in the analysis of the tissue heating process is to estimate the degree of its destruction (Abraham and Sparrow, 2007; Henriques, 1947; Jasiński, 2018). The Arrhenius injury integral is the most frequently applied tool for this purpose, although other models (e.g. thermal dose) are also used (Mochnacki and Piasecka-Belkhayat, 2013). The Arrhenius scheme assumes the exponential dependence between temperature and the degree of tissue destruction. Furthermore, it refers only to the irreversible tissue damage, however, there are models which allow one to take into account the withdrawal of tissue injury in the case of temporary, small local increasing of temperature (Jasiński, 2014, 2018).

The purpose of this paper is to analyse the phenomena occurring in the laser-treated soft tissue wherein thermophysical and optical parameters are defined and treated as directed interval numbers. The analysis is based on the bioheat transfer equation in the Pennes formulation, whereas, to describe the light distribution in tissue the steady-state diffuse approximation is used. The degree of tissue destruction is also estimated by the use of the Arrhenius scheme. At the stage of numerical realisation, the interval finite difference method is used (Majchrzak and Mochnacki, 2016, 2017; Mochnacki and Suchy, 1995).

\section{Formulation of the problem}

A transient heat transfer in biological tissue is described by the Pennes equation. The interval form of this equation can be expressed in the form (Abraham and Sparrow, 2007; Jankowska and Sypniewska-Kaminska, 2012; Jasiński, 2014; Paruch, 2014)

$$
\mathbf{x} \in \Omega: \quad \bar{c} \frac{\partial \bar{T}}{\partial t}=\bar{\lambda} \nabla^{2} \bar{T}+\bar{Q}_{p e r f}+\bar{Q}_{m e t}+\bar{Q}_{\text {las }}
$$

where $\bar{\lambda}\left[\mathrm{Wm}^{-1} \mathrm{~K}^{-1}\right]$ is the interval thermal conductivity, $\bar{c}\left[\mathrm{Jm}^{-3} \mathrm{~K}^{-1}\right]$ is the interval volumetric specific heat, $\bar{Q}_{\text {perf }}, \bar{Q}_{m e t}$ and $\bar{Q}_{\text {las }}\left[\mathrm{Wm}^{-3}\right]$ are the interval internal heat sources containing 
information connected with the perfusion, metabolism and laser irradiation, respectively, while $\bar{T}=\bar{T}(\mathbf{x}, t)[\mathrm{K}]$ is the interval temperature.

In this paper, the $2 \mathrm{D}$ domain of homogeneous biological tissue of a rectangular shape $\Omega$ subjected to the laser action is considered (Fig. 1).

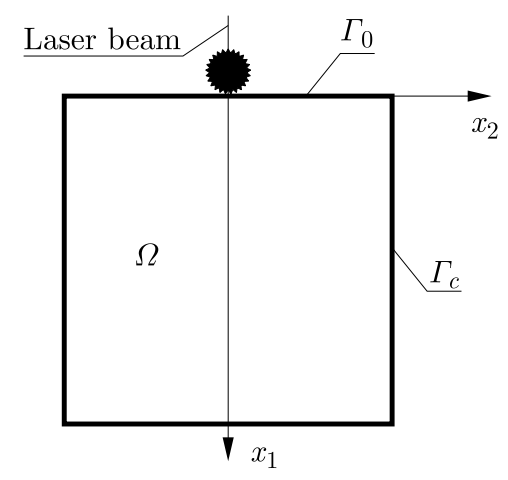

Fig. 1. The domain considered

Equation (2.1) is supplemented by the Robin condition assumed on the external boundary of tissue $\Gamma_{0}$, which is subjected to laser irradiation while on the remaining parts of the boundary $\Gamma_{c}$ the no-flux condition is accepted

$$
\begin{array}{ll}
\mathbf{x} \in \Gamma_{0}: & \bar{q}(\mathbf{x}, t)=\alpha\left(\bar{T}-T_{a m b}\right) \\
\mathbf{x} \in \Gamma_{c}: & \bar{q}(\mathbf{x}, t)=0
\end{array}
$$

where $\alpha\left[\mathrm{Wm}^{-2} \mathrm{~K}^{-1}\right]$ is the convective heat transfer coefficient and $T_{a m b}$ is temperature of the surroundings. The initial distribution of temperature is also known

$$
\mathbf{x} \in \Omega, t=0: \quad T(\mathbf{x}, t)=T_{p}
$$

In the current work, the metabolic heat source $\bar{Q}_{m e t}\left[\mathrm{Wm}^{-3}\right]$ is assumed as a constant interval parameter while the perfusion heat source is described by the formula

$$
\bar{Q}_{p e r f}(\mathbf{x}, t)=c_{B} \bar{w}\left[T_{B}-\bar{T}(\mathbf{x}, t)\right]
$$

where $\bar{w}\left[\mathrm{~s}^{-1}\right]$ is the interval perfusion coefficient, $c_{B}\left[\mathrm{Jm}^{-3} \mathrm{~K}^{-1}\right]$ is the volumetric specific heat of blood and $T_{B}$ corresponds to the arterial temperature (Abraham and Sparrow, 2007; Mochnacki and Piasecka, 2013).

The source function $\bar{Q}_{\text {las }}$ connected with the laser heating is defined as follows (Jasiński et al., 2016)

$$
\bar{Q}_{l a s}(\mathbf{x}, t)=\bar{\mu}_{a} \bar{\phi}(\mathbf{x}) p(t)
$$

where $\bar{\mu}_{a}\left[\mathrm{~m}^{-1}\right]$ is the interval absorption coefficient, $\bar{\phi}(\mathbf{x})\left[\mathrm{Wm}^{-2}\right]$ is the interval total light fluence rate and $p(t)$ is the function equal to 1 when the laser is on and equal to 0 when the laser is off.

The total interval light fluence rate $\bar{\phi}$ is the sum of the interval collimated part $\bar{\phi}_{c}$ and diffuse part $\bar{\phi}_{d}$ (Banerjee and Sharma, 2010; Dombrovsky et al., 2013)

$$
\bar{\phi}(\mathbf{x})=\bar{\phi}_{c}(\mathbf{x})+\bar{\phi}_{d}(\mathbf{x})
$$

The collimated fluence rate is given as (Jasiński et al., 2016)

$$
\bar{\phi}_{c}(\mathbf{x})=\phi_{0} \exp \left(-\frac{2 x_{2}^{2}}{r^{2}}\right) \exp \left(-\bar{\mu}_{t}^{\prime} x_{1}\right)
$$


where $\phi_{0}\left[\mathrm{Wm}^{-2}\right]$ is the surface irradiance of laser, $r$ is the radius of the laser beam and $\bar{\mu}_{t}^{\prime}\left[\mathrm{m}^{-1}\right]$ is the interval attenuation coefficient defined as (Banerjee and Sharma, 2010)

$$
\bar{\mu}_{t}^{\prime}=\bar{\mu}_{a}+\bar{\mu}_{s}^{\prime}=\bar{\mu}_{a}+(1-g) \bar{\mu}_{s}
$$

where $\bar{\mu}_{s}$ and $\bar{\mu}_{s}^{\prime}\left[\mathrm{m}^{-1}\right]$ are the interval scattering coefficient and the effective scattering coefficient, respectively, while $g$ is the anisotropy factor.

To determine the interval diffuse fluence rate $\bar{\phi}_{d}$, the steady-state optical diffusion equation should be solved (Dombrovsky et al., 2013; Welch, 2011)

$$
\mathbf{x} \in \Omega: \quad \bar{D} \nabla^{2} \bar{\phi}_{d}(\mathbf{x})-\bar{\mu}_{a} \bar{\phi}_{d}(\mathbf{x})+\bar{\mu}_{s}^{\prime} \bar{\phi}_{c}(\mathbf{x})=0
$$

where

$$
\bar{D}=\frac{1}{3\left[\bar{\mu}_{a}+(1-g) \bar{\mu}_{s}\right]}=\frac{1}{3 \bar{\mu}_{t}^{\prime}}
$$

is the interval diffusion coefficient.

Equation (2.9) is supplemented by the boundary conditions on the boundaries $\Gamma_{0}$ and $\Gamma_{c}$

$$
\mathbf{x} \in \Gamma_{0}, \Gamma_{c}: \quad-\bar{D} \mathbf{n} \cdot \nabla \bar{\phi}_{d}(\mathbf{x})=\frac{\bar{\phi}_{d}(\mathbf{x})}{2}
$$

where $\mathbf{n}$ is the outward unit normal vector.

Damage of biological tissue resulting from temperature elevation is modelled by the Arrhenius injury integral, and its interval version considered in this paper is defined as (Abraham and Sparrow, 2007; Fasano et al., 2010; Henriques, 1947; Mochnacki and Piasecka-Belkhayat, 2013)

$$
\bar{\Psi}\left(\mathbf{x}, t^{F}\right)=\int_{0}^{t^{F}} P \exp \left[-\frac{E}{R \bar{T}(\mathbf{x}, t)}\right] d t
$$

where $R\left[\mathrm{~J} \mathrm{~mole}^{-1} \mathrm{~K}^{-1}\right]$ is the universal gas constant, $E\left[\mathrm{~J} \mathrm{~mole}^{-1}\right]$ is the activation energy and $P\left[\mathrm{~s}^{-1}\right]$ is the pre-exponential factor while $\left[0, t^{F}\right]$ is the considered time interval. The criterion for tissue necrosis is $\Psi(\mathbf{x}) \geqslant 1$.

\section{Method of solution}

In this paper, both analyzed equations: the Pennes equation and the steady-state optical diffusion equation have been solved using the interval finite difference method (Mochnacki and Piasecka-Belkhayat, 2013). The information about the directed interval arithmetic are presented in (Dawood, 2011; Piasecka-Belkhayat, 2011; Popova, 2011).

In order to determine the function $\bar{Q}_{\text {las }}$ at the internal node $(i, j)$ (cf. equation (2.5)), steady-state optical diffusion equation (2.9) is solved. The uniform differential grid of dimension $2 n \times 2 n$ with spacing $h / 2$ is used here (Fig. 2). Using such a differential grid, it is easier to take into account boundary conditions (2.11), because a part of the nodes is located exactly on the boundary $\Gamma_{0}$ and $\Gamma_{c}$. In addition, one can distinguish common grid nodes for the temperature and diffuse fluence rate.

The following differential quotients are used

$$
\left(\frac{\partial^{2} \bar{\phi}_{d}}{\partial x_{1}^{2}}\right)_{i, j}=\frac{\bar{\phi}_{d i+1, j}-2 \bar{\phi}_{d i, j}+\bar{\phi}_{d i-1, j}}{(h / 2)^{2}} \quad\left(\frac{\partial^{2} \bar{\phi}_{d}}{\partial x_{2}^{2}}\right)_{i, j}=\frac{\bar{\phi}_{d i, j+1}-2 \bar{\phi}_{d i, j}+\bar{\phi}_{d i, j-1}}{(h / 2)^{2}}
$$



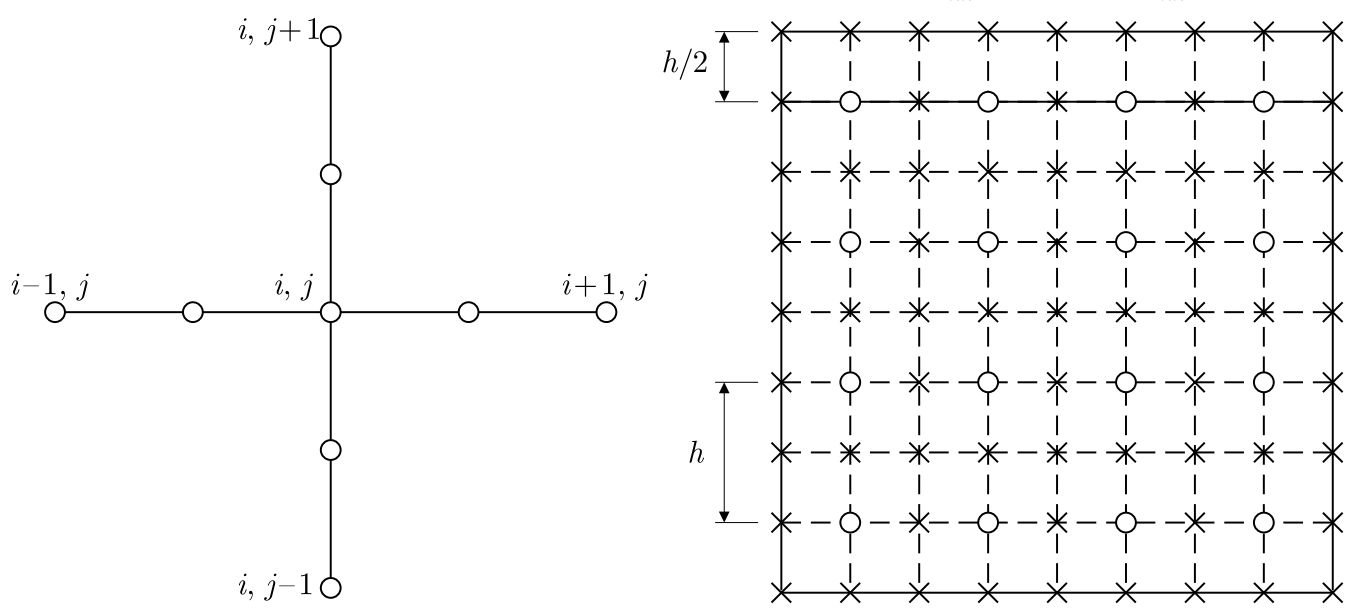

Fig. 2. Five-point stencil and differential grid

and then the approximate form of equation for the internal node $(i, j)(i=1,2, \ldots, 2 n-1$, $j=1,2, \ldots, 2 n-1)$ is as follows

$$
\bar{\phi}_{d i, j}=\bar{C}_{1} \bar{\Phi}_{d i, j}+\bar{C}_{2} \bar{\phi}_{c i, j}
$$

where (superscripts "-" and "+" denote the beginning and the end of the interval, respectively)

$$
\begin{aligned}
& \bar{\Phi}_{d i, j}=\bar{\phi}_{d i-1, j}+\bar{\phi}_{d i+1, j}+\bar{\phi}_{d i, j+1}+\bar{\phi}_{d i, j-1} \\
& \left.\quad=\phi_{d i-1, j}^{-}+\phi_{d i+1, j}^{-}+\phi_{d i, j+1}^{-}+\phi_{d i, j-1}^{-}, \phi_{d i-1, j}^{+}+\phi_{d i+1, j}^{+}+\phi_{d i, j+1}^{+}+\phi_{d i, j-1}^{+}\right]
\end{aligned}
$$

while

$$
\begin{aligned}
\bar{C}_{1} & =\frac{4 \bar{D}}{16 \bar{D}+h^{2} \bar{\mu}_{a}}=\frac{4\left[D^{-}, D^{+}\right]}{16\left[D^{-}, D^{+}\right]+h^{2}\left[\mu_{a}^{-}, \mu_{a}^{+}\right]}=\frac{\left[4 D^{-}, 4 D^{+}\right]}{\left[16 D^{-}+h^{2} \mu_{a}^{-}, 16 D^{+}+h^{2} \mu_{a}^{+}\right]} \\
& =\left[\frac{4 D^{-}}{16 D^{-}+h^{2} \mu_{a}^{-}}, \frac{4 D^{+}}{16 D^{+}+h^{2} \mu_{a}^{+}}\right] \\
\bar{C}_{2} & =\frac{\bar{\mu}_{s}^{\prime} h^{2}}{16 \bar{D}+\bar{\mu}_{a} h^{2}}=\frac{h^{2}\left[\mu_{s}^{\prime}, \mu_{s}^{\prime}\right]}{16\left[D^{-}, D^{+}\right]+h^{2}\left[\mu_{a}^{-}, \mu_{a}^{+}\right]}=\frac{\left[h^{2} \mu_{s}^{\prime}, h^{2} \mu_{s}^{\prime+}\right]}{\left[16 D^{-}+h^{2} \mu_{a}^{-}, 16 D^{+}+h^{2} \mu_{a}^{+}\right]} \\
& =\left[\frac{h^{2} \mu_{s}^{\prime}}{16 D^{-}+h^{2} \mu_{a}^{-}}, \frac{h^{2} \mu_{s}^{\prime}}{16 D^{+}+h^{2} \mu_{a}^{+}}\right]
\end{aligned}
$$

while for boundary nodes (cf. equation (2.11))

$$
\begin{aligned}
& \bar{D} \frac{\bar{\phi}_{d 1, j}-\bar{\phi}_{d 0, j}}{h / 2}=\frac{1}{2} \bar{\phi}_{d 0, j} \quad \rightarrow \quad \bar{\phi}_{d 0, j}=\bar{C}_{3} \bar{\phi}_{d 1, j} \\
& -\bar{D} \frac{\bar{\phi}_{d n, j}-\bar{\phi}_{d n-1, j}}{h / 2}=\frac{1}{2} \bar{\phi}_{d n, j} \quad \rightarrow \quad \bar{\phi}_{d n, j}=\bar{C}_{3} \bar{\phi}_{d n-1, j} \\
& \bar{D} \frac{\bar{\phi}_{d i, j}-\bar{\phi}_{d i, 0}}{h / 2}=\frac{1}{2} \bar{\phi}_{d i, 0} \quad \rightarrow \quad \bar{\phi}_{d i, 0}=\bar{C}_{3} \bar{\phi}_{d i, 1} \\
& -\bar{D} \frac{\bar{\phi}_{d i, n}-\bar{\phi}_{d i, n-1}}{h / 2}=\frac{1}{2} \bar{\phi}_{d i, n} \quad \rightarrow \quad \bar{\phi}_{d i, n}=\bar{C}_{3} \bar{\phi}_{d i, n-1}
\end{aligned}
$$

where

$$
\bar{C}_{3}=\frac{4 \bar{D}}{4 \bar{D}+h}=\frac{4\left[D^{-}, D^{+}\right]}{4\left[D^{-}, D^{+}\right]+h^{2}}=\frac{\left[4 D^{-}, 4 D^{+}\right]}{\left[4 D^{-}+h^{2}, 4 D^{+}+h^{2}\right]}=\left[\frac{4 D^{-}}{4 D^{-}+h^{2}}, \frac{4 D^{+}}{4 D^{+}+h^{2}}\right]
$$


It should be pointed out that the system of equations (3.2) is solved using the iterative method.

The differential quotients approximating the derivatives appearing in the Pennes equation (2.1) are defined in a similar, as previously, manner

$$
\begin{aligned}
& \left(\frac{\partial^{2} \bar{T}}{\partial x_{1}^{2}}\right)_{i, j}=\frac{\bar{T}_{i+1, j}-2 \bar{T}_{i, j}+\bar{T}_{i-1, j}}{h^{2}} \quad\left(\frac{\partial^{2} \bar{T}}{\partial x_{2}^{2}}\right)_{i, j}=\frac{\bar{T}_{i, j+1}-2 \bar{T}_{i, j}+\bar{T}_{i, j-1}}{h^{2}} \\
& \frac{\partial \bar{T}}{\partial t}=\frac{\bar{T}_{i, j}^{f}-\bar{T}_{i, j}^{f-1}}{\Delta t}
\end{aligned}
$$

where $\Delta t$ denotes the time step while $f-1$ and $f$ are the subsequent time levels.

By introducing this formulas into (2.1), and after some mathematical operations for the internal nodes $(i=1,2, \ldots, n, j=1,2, \ldots, n)$, one obtains

$$
\bar{T}_{i, j}^{f}=\bar{A}_{2} \bar{T}_{i, j}^{f-1}+\bar{A}_{1} \bar{\Upsilon}_{i, j}^{f-1}+\bar{A}_{3}
$$

where

$$
\begin{gathered}
\bar{\Upsilon}_{i, j}^{f-1}=\bar{T}_{i+1, j}^{f-1}+\bar{T}_{i-1, j}^{f-1}+\bar{T}_{i, j+1}^{f-1}+\bar{T}_{i, j-1}^{f-1}=\left[\left(T_{i+1, j}^{f-1}\right)^{-}+\left(T_{i-1, j}^{f-1}\right)^{-}+\left(T_{i, j+1}^{f-1}\right)^{-}+\left(T_{i, j-1}^{f-1}\right)^{-},\right. \\
\left.\left(T_{i+1, j}^{f-1}\right)^{+}+\left(T_{i-1, j}^{f-1}\right)^{+}+\left(T_{i, j+1}^{f-1}\right)^{+}+\left(T_{i, j-1}^{f-1}\right)^{+}\right]
\end{gathered}
$$

while

$$
\begin{aligned}
\bar{A}_{1} & =\frac{\bar{\lambda} \Delta t}{\bar{c} h^{2}}=\frac{\Delta t\left[\lambda^{-}, \lambda^{+}\right]}{h^{2}\left[c^{-}, c^{+}\right]}=\frac{\left[\Delta t \lambda^{-}, \Delta t \lambda^{+}\right]}{\left[h^{2} c^{-}, h^{2} c^{+}\right]}=\left[\frac{\Delta t \lambda^{-}}{h^{2} c^{-}}, \frac{\Delta t \lambda^{+}}{h^{2} c^{+}}\right] \\
\bar{A}_{2} & =1-\bar{A}_{1}-\frac{\bar{w} c_{B} \Delta t}{\bar{c}}=\left[1-A_{1}^{-}, 1-A_{1}^{+}\right]-\frac{c_{B} \Delta t\left[w^{-}, w^{+}\right]}{\left[c^{-}, c^{+}\right]}=\left[1-A_{1}^{-}, 1-A_{1}^{+}\right] \\
& -\left[\frac{c_{B} \Delta t w^{-}}{c^{-}}, \frac{c_{B} \Delta t w^{+}}{c^{+}}\right]=\left[1-A_{1}^{-}-\frac{c_{B} \Delta t w^{-}}{c^{-}}, 1-A_{1}^{+}-\frac{c_{B} \Delta t w^{+}}{c^{+}}\right] \\
\bar{A}_{3} & =\frac{\Delta t}{\bar{c}}\left(\bar{w} c_{B} T_{B}+\bar{Q}_{\text {met }}+\bar{Q}_{\text {las }}\right)=\frac{\Delta t}{\left[c^{-}, c^{+}\right]}\left(c_{B} T_{B}\left[w^{-}, w^{+}\right]+\left[Q_{\text {met }}^{-}, Q_{\text {met }}^{-}\right]+\left[Q_{\text {las }}^{-}, Q_{\text {las }}^{-}\right]\right) \\
& =\left[\frac{\Delta t\left(c_{B} T_{B} w^{-}+Q_{\text {met }}^{-}+Q_{\text {las }}^{-}\right)}{c^{-}}, \frac{\Delta t\left(c_{B} T_{B} w^{+}+Q_{\text {met }}^{+}+Q_{\text {las }}^{+}\right)}{c^{+}}\right]
\end{aligned}
$$

The "boundary" nodes are located at the distance $0.5 h$ from the boundary of the domain. This approach gives a better approximation of the Neumann and Robin boundary conditions, but the final form of the interval FDM equation for the boundary nodes are obtained in similar way. More details of this approach can be found in (Jasiński et al., 2016; Majchrzak and Mochnacki, 2016, 2017).

On the basis of equation (3.8), the temperature at the node $(i, j)$ for the time level can be found on the assumption that the stability condition for an explicit differential scheme is fulfilled (Mochnacki and Suchy, 1995).

\section{Results of computations}

At the stage of numerical computations, a 2D homogeneous tissue domain of size $4 \times 4 \mathrm{~cm}$ during laser irradiation (Fig. 1) has been considered. The uniform differential grid with $40 \times 40$ nodes is introduced (Fig. 2). As has been already mentioned, the values of the effective scattering coefficient are different for the native and thermally-damaged (denaturated) tissue, so the two 
simulations have been conducted (for $\mu_{s}^{\prime}=\mu_{s \text { nat }}^{\prime}$ and $\mu_{s}^{\prime}=\mu_{s d e n}^{\prime}$ ). The optical $\left(\mu_{a}, \mu_{s}^{\prime}\right.$ ) and thermophysical $\left(\lambda, c, w, Q_{m e t}\right)$ parameters of the tissue are assumed as the directed interval numbers in form (Dombrovsky et al., 2013; Popova, 2011)

$$
\bar{p}=[p-0.025 p, p+0.025 p]
$$

where $p$ denotes the parameter.

The following data have been assuming in calculations: $\lambda=0.609 \mathrm{Wm}^{-1} \mathrm{~K}^{-1}$, $c=4.18 \mathrm{MJm}^{-3} \mathrm{~K}^{-1}, w=0.00125 \mathrm{~s}^{-1}, \mu_{a}=40 \mathrm{~m}^{-1}, \mu_{\text {snat }}^{\prime}=1000 \mathrm{~m}^{-1}, \mu_{\text {sden }}^{\prime}=4000 \mathrm{~m}^{-1}$, $Q_{\text {met }}=245 \mathrm{Wm}^{-3}, c_{B}=3.9962 \mathrm{MJm}^{-3} \mathrm{~K}^{-1}, T_{B}=37^{\circ} \mathrm{C}, P=3.1 \cdot 10^{98} \mathrm{~s}^{-1}$, $E=6.27 \cdot 10^{5} \mathrm{~J} \mathrm{~mole}^{-1}, R=8.314 \mathrm{~J} \mathrm{~mole}^{-1} \mathrm{~K}^{-1}, \phi_{0}=3 \cdot 10^{5} \mathrm{Wcm}^{-2}, d=2 \mathrm{~mm}, t_{\text {exp }}=25 \mathrm{~s}$, $\alpha=10 \mathrm{Wm}^{-2} \mathrm{~K}^{-1}, T_{a m b}=20^{\circ} \mathrm{C}, T_{p}=37^{\circ} \mathrm{C}, \Delta t=1 \mathrm{~s}$.

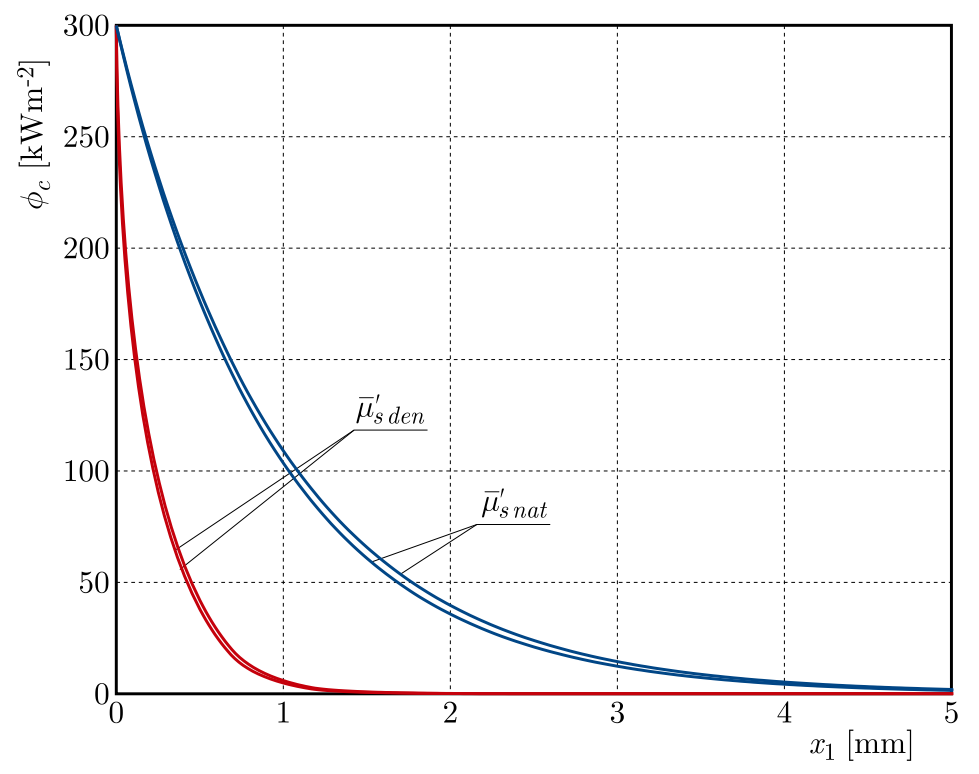

Fig. 3. Distribution of the interval collimated fluence rate $\bar{\phi}_{c}\left(x_{2}=0\right)$

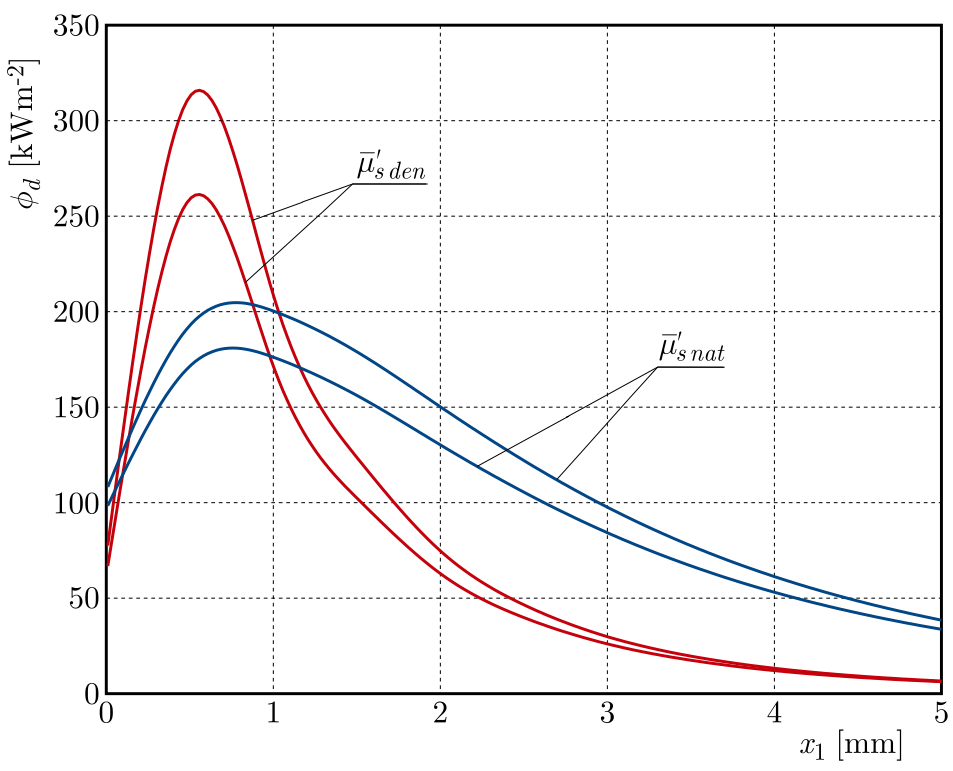

Fig. 4. Distribution of the interval diffuse fluence rate $\bar{\phi}_{d}\left(x_{2}=0\right)$

Figures 3-5 are associated with the light fluence distribution in the domain considered. Figure 3 presents the distribution of the interval collimated fluence rate $\bar{\phi}_{c}$ calculated on the 
basis of equation (2.7) while in Fig. 4 the distribution of the interval diffuse fluence rate $\bar{\phi}_{d}$ resulting from steady-state optical diffusion equation (2.9) is presented. The distribution of the interval diffuse fluence rate $\bar{\phi}_{d}$ is also presented in Fig. 5. It is visible that the area of scattering is larger in the case of calculation with $\bar{\mu}_{s \text { nat }}^{\prime}$ although the values of $\bar{\phi}_{d}$ are lower than in the case of calculation with $\bar{\mu}_{s d e n}^{\prime}$.
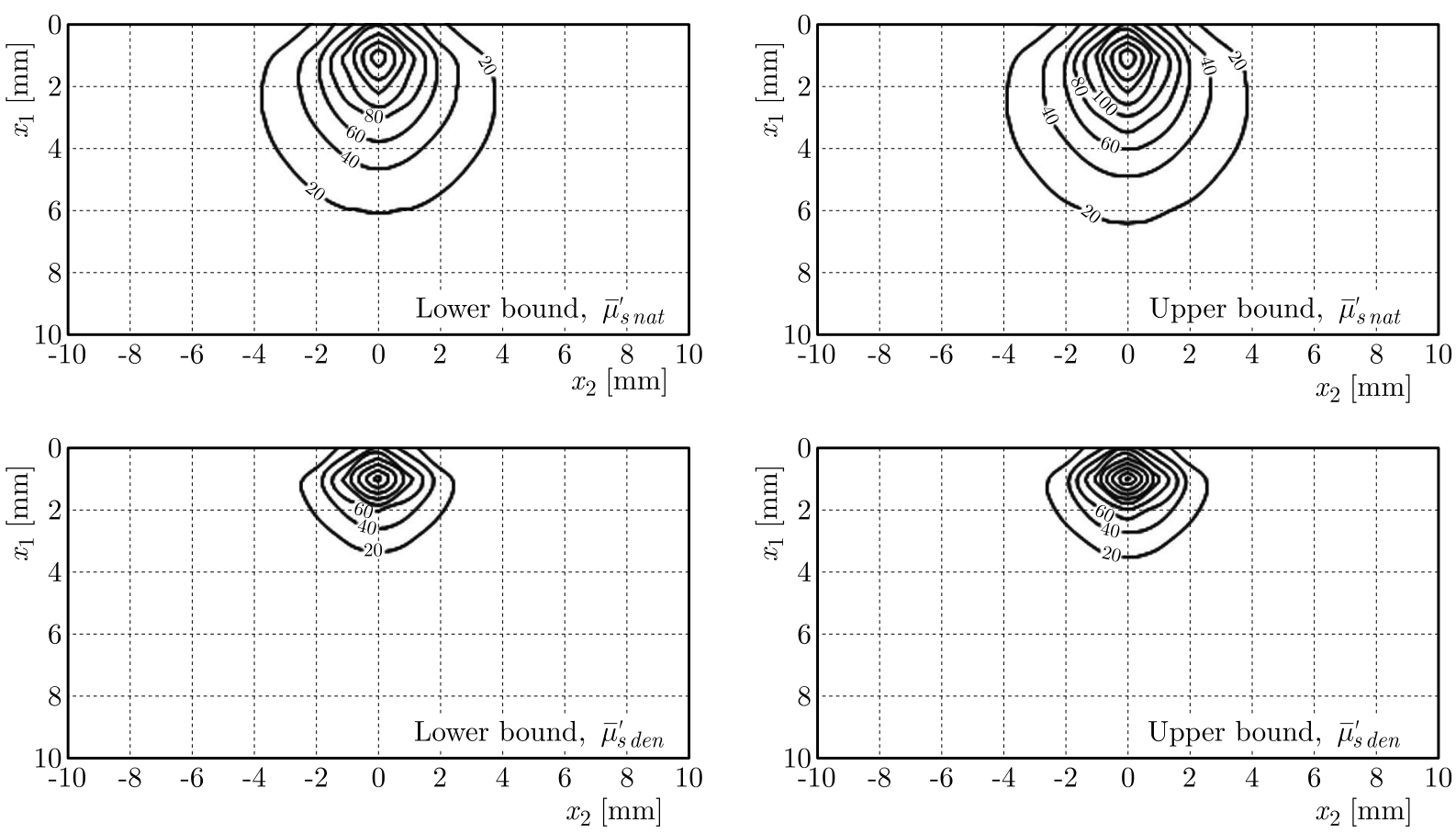

Fig. 5. Distribution of the interval diffuse fluence rate $\bar{\phi}_{d}\left[\mathrm{kWm}^{-2}\right]$

The next figure is associated with the tissue temperature. In Fig. 6, the interval tissue temperature history at the node $N_{0}(0,0)$ (Fig. 1) obtained for the effective scattering coefficient of native and denaturated tissue is presented. As can be seen, wider temperature intervals are obtained in the case of denaturated tissue.

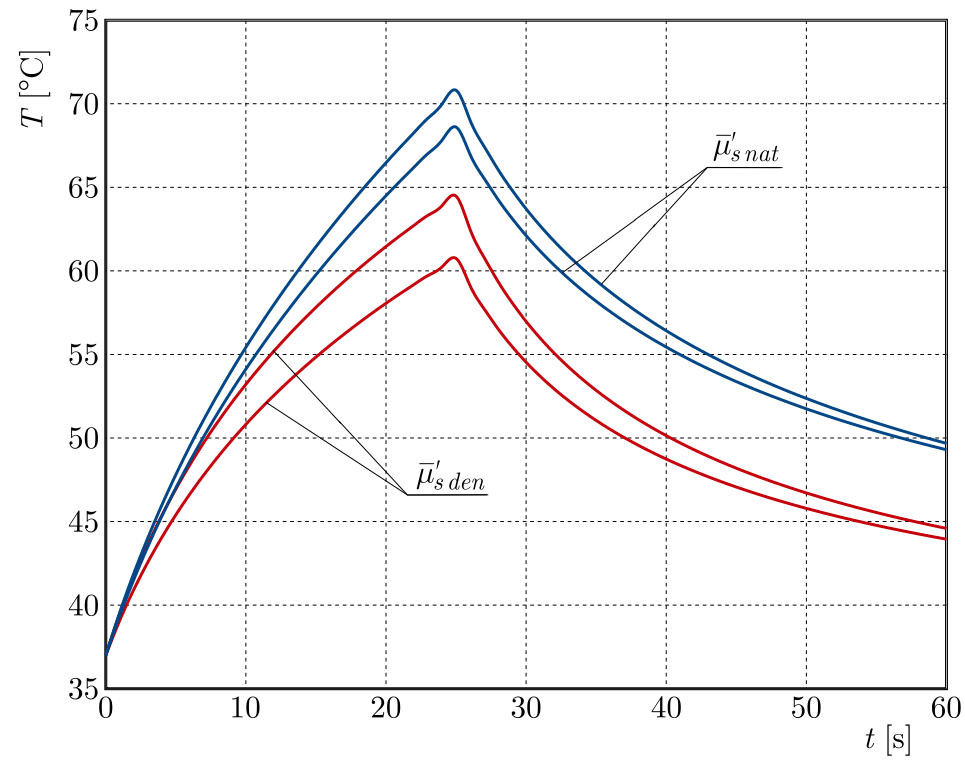

Fig. 6. History of interval tissue temperature at the node $N_{0}$ 


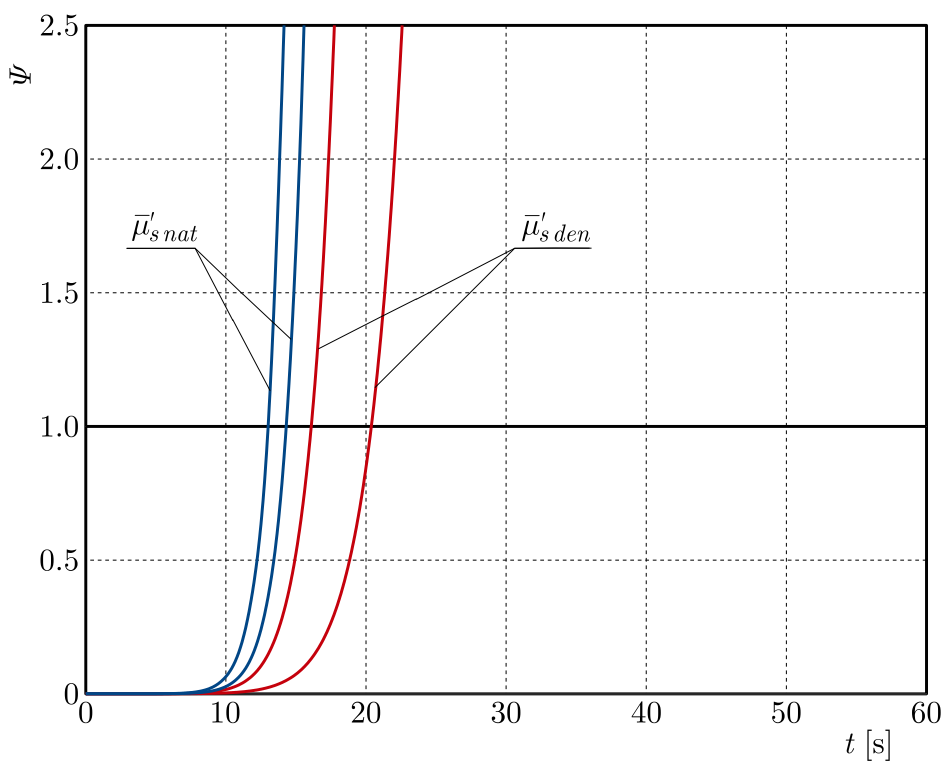

Fig. 7. History of the interval Arrhenius integral at the node $N_{0}$
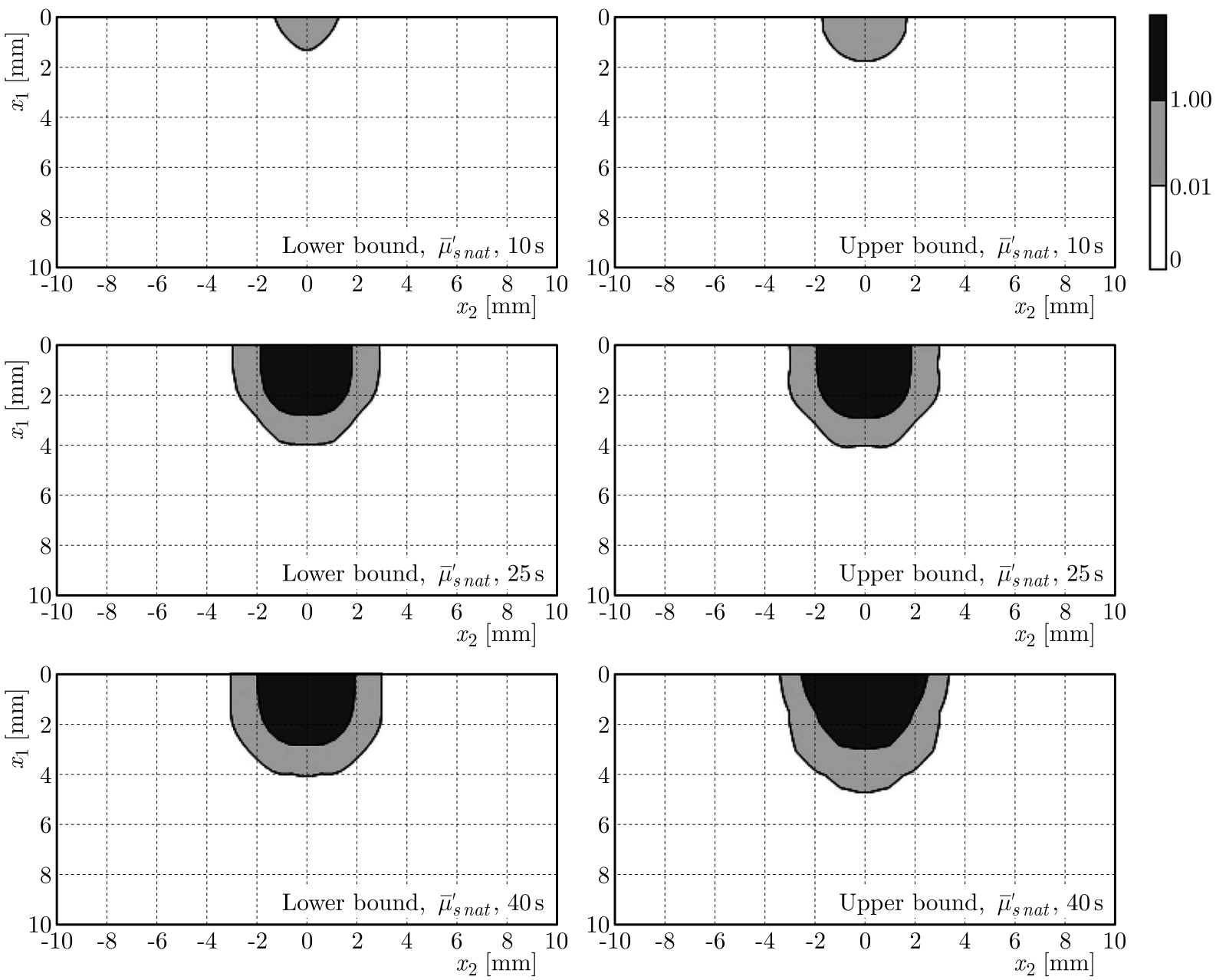

Fig. 8. Distributions of the interval Arrhenius injury integral for native tissue $\left(\bar{\mu}_{s}^{\prime}=\bar{\mu}_{s \text { nat }}^{\prime}\right)$ 
In Fig. 7, the history of the interval Arrhenius integral at the node $N_{0}$ is shown. As expected, due to the wider intervals obtained in the calculations with $\bar{\mu}_{s d e n}^{\prime}$, wider intervals for the interval Arrhenius integral are also obtained for this variant of calculations. It is visible that tissue destruction occurs first in the case of native tissue - the injury integral reaches the necrosis criterion $(\Psi \geqslant 1)$ in the time interval $[14,15] \mathrm{s}$ while for calculation with $\bar{\mu}_{s d e n}^{\prime}$ this criterion is reached in the time interval $[17,21] \mathrm{s}$.

The results associated with the tissue destruction are also presented in Figs. 8 and 9. In both figures, the interval injury integral distributions for selected time steps are presented. The white zone in these figures refers to the values of the injury integral below 0.01 (thermally untouched tissue), the grey zone refers to the values $0.01<\Psi<1$, so it is a partial damage area, and the black zone illustrates the area in which the Arrhenius integral achieved the criterion of tissue necrosis. In both cases, the coagulation zones obtained for the lower and upper bounds of intervals are slightly different, however, in the case of destructed tissue the differences are bigger.
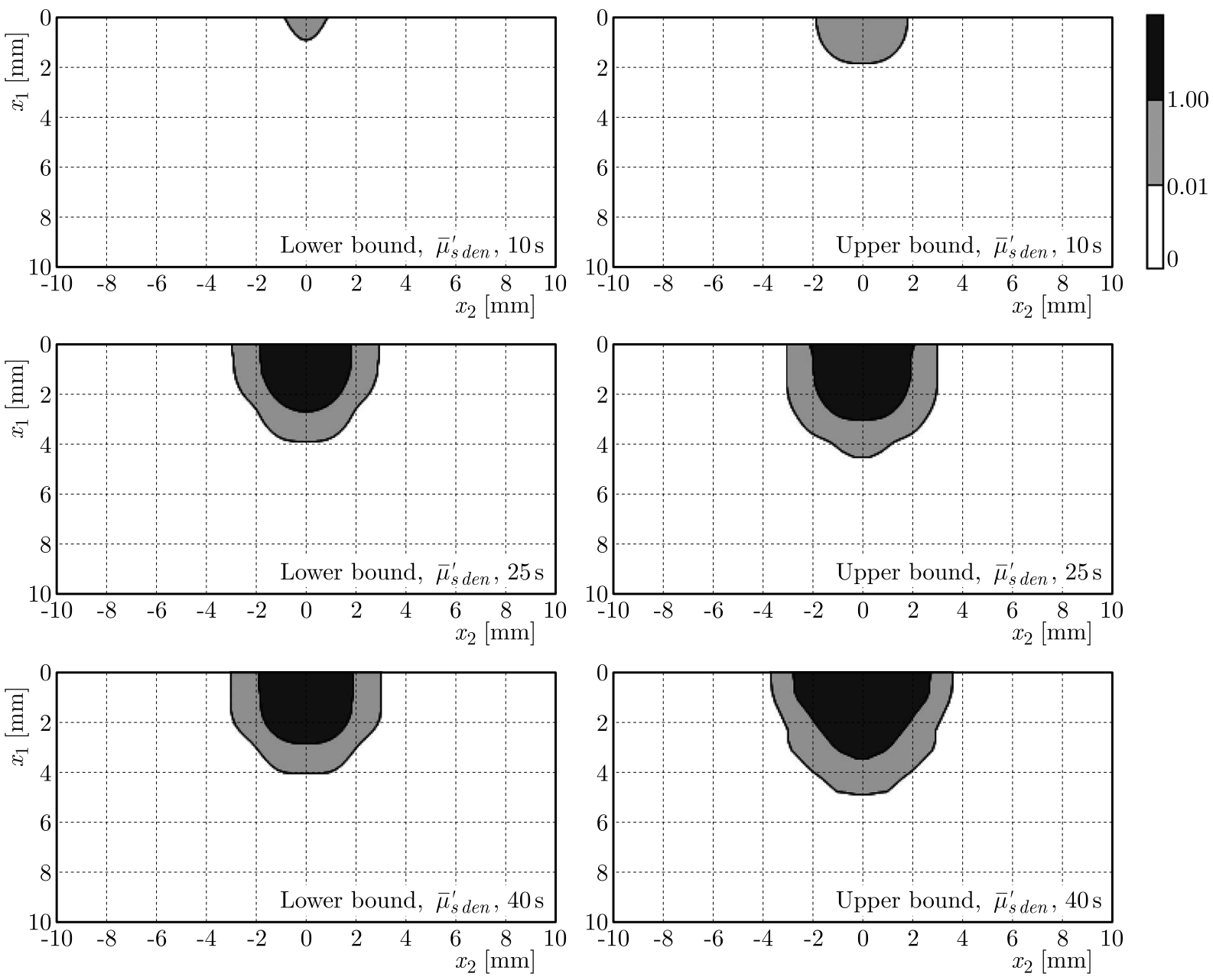

Fig. 9. Distributions of the interval Arrhenius injury integral for denaturated tissue $\left(\bar{\mu}_{s}^{\prime}=\bar{\mu}_{s d e n}^{\prime}\right)$

\section{Discussion}

As has been mentioned before, the reason for the application of the directed interval arithmetic was to take into account the inaccuracies of estimation of biological tissue parameters and their effect on thermal damage to the tissue. It can be seen that the results associated with the tissue 
damage are quite reasonable for the assumed width of interval parameters. The differences between the ranges of coagulation zones (Figs. 8 and 9) obtained for both assumed values of the interval effective scattering coefficient $\bar{\mu}_{s \text { nat }}^{\prime}$ and $\bar{\mu}_{s d e n}^{\prime}$ are clearly visible. On the other hand, the differences between the lower and upper bounds of the interval Arrhenius integral for both cases of $\bar{\mu}_{s}^{\prime}$ are not very big.

Of course, thermal damage can also expand after the impulse causing the temperature elevation ceases, what happens also in the analysed cases for $t>t_{\text {exp }}$, and the thermal injury is fully formed in less than $40 \mathrm{~s}$.

Application of the Arrhenius integral also allows one to estimate the time after which the given point of the domain considered is thermally damaged, i.e. the value $\bar{\Psi}$ reaches the necrosis criterion. The results of this type are shown in Fig. 7. These results allow one to estimate the time of tissue damage in a few second intervals - at the node $N_{0}$ for the calculation with $\bar{\mu}_{s \text { nat }}^{\prime}$ $[14,15] \mathrm{s}$ while for the calculation with $\bar{\mu}_{s d e n}^{\prime}[17,21] \mathrm{s}$. Such width of intervals can be accepted as useful information from a practical point of view.

It should be pointed out that, in some cases, it is justified to assume a more restrictive necrosis criterion, i.e. $\Psi(\mathbf{x}) \geqslant 4.6$ which corresponds to $99 \%$ of dead cells (e.g. laser cancer therapy). This criterion is also fulfilled at the node $N_{0}$ for the assumed width of the parameter intervals - for calculation with $\bar{\mu}_{\text {snat }}^{\prime}[15,17] \mathrm{s}$, for calculation with $\bar{\mu}_{s d e n}^{\prime}[19,25] \mathrm{s}$. These width of intervals are a still reasonable outcome.

One should also note that an increase in the intervals for optical and thermophysical parameters leads to an increase in temperature intervals, which are the basic value in calculations of the interval Arrhenius integral. This, in turn, may lead to a situation in which the lower bound of the interval injury integral for a selected point of the domain considered would be below the threshold of necrosis, whereas the upper bound would exceed this threshold.

\section{Conclusions}

In the paper, the analysis of the thermal damage of biological tissue subjected to laser impulse has been presented, whereas, the optical and thermophysical parameters of tissue have been treated as the directed interval numbers. That concerned two optical parameters: absorption coefficient $\bar{\mu}_{a}$, effective scattering coefficient of tissue $\bar{\mu}_{s}^{\prime}$, and four thermophysical parameters: thermal conductivity $\bar{\lambda}$, volumetric specific heat $\bar{c}$, perfusion coefficient $\bar{w}$ and metabolic heat source $\bar{Q}_{m e t}$. The combined effect of those parameters has been considered, although it is known that not all of these parameters (or actual changes in parameters values) have the same influence on the temperature level and, as a result, on the estimated value of tissue damage. It has been described in numerous works related to e.g. sensitivity analysis (Majchrzak and Mochnacki, 2017; Mochnacki and Ciesielski, 2016). Of course, interval analysis for the individual parameters of tissue is also possible to perform.

The directed interval arithmetic has already been effectively applied to the modelling of bioheat transfer problems. Most of the works were based on the Pennes equation, although it is possible to use a different bioheat transfer equation (Mochnacki and Piasecka-Belkhayat, 2013). Of particular interest here is the use of the GDPL equation based on the theory of porous bodies which binds the process of heat transfer with the inner tissue structure (Majchrzak and Mochnacki, 2017; Majchrzak et al., 2015).

The modelling of the tissue thermal damage process also includes a number of other problems, e.g. methods of taking into account changes in parameter values caused by the degree of tissue damage (Jasiński, 2015, 2018). Further work related to the application of directed interval arithmetic in this area could concern these issues or the problems related with the use of interval numbers in other algorithms related to thermal damage (Jasiński, 2015; Mochnacki and 
Piasecka, 2013). Because the interval arithmetic is a method developed by mathematicians in order to put bounds on rounding errors and measurement errors in mathematical computation, it could also be a useful approach to the modelling of various problems of bioheat transfer.

\section{Acknowledgements}

The research has been funded from the projects of Silesian University of Technology, Faculty of Mechanical Engineering.

\section{References}

1. Abraham J.P., Sparrow E.M., 2007, A thermal-ablation bioheat model including liquid-to-vapor phase change, pressure- and necrosis-dependent perfusion, and moisture-dependent properties, International Journal of Heat and Mass Transfer, 50, 13-14, 2537-2544

2. BanerJee S., Sharma S.K., 2010, Use of Monte Carlo simulations for propagation of light in biomedical tissues, Applied Optics, 49, 4152-4159

3. Dawood H., 2011, Theories of Interval Arithmetic: Mathematical Foundations and Applications, LAP LAMBERT Academic Publishing GmbH \& Co., Germany

4. Dombrovsky L.A., Baillis D., 2010, Thermal Radiation in Disperse Systems: An Engineering Approach, Begell House, New York

5. Dombrovsky L.A., Randrianalisoa J.H., Lipinski W., Timchenko V., 2013, Simplified approaches to radiative transfer simulations in laser induced hyperthermia of superficial tumors, Computational Thermal Sciences, 5, 6, 521-530

6. Fasano A., Hömberg D., Naumov D., 2010, On a mathematical model for laser-induced thermotherapy, Applied Mathematical Modelling, 34, 12, 3831-3840

7. Gajda K., Marciniak A., Szyszka B., 2000, Three- and four-stage implicit interval methods of Runge-Kutta type, Computational Methods in Science and Technology, 6, 41-59

8. Hansen E., Walster G.W., 2004, Global Optimization Using Interval Analysis, Marcell Dekker, New York

9. Henriques F.C., 1947, Studies of thermal injuries, V. The predictability and the significance of thermally induced rate process leading to irreversible epidermal injury, Journal of Pathology, 23, 489-502

10. Jacques S.L., Pogue B.W., 2008, Tutorial on diffuse light transport, Journal of Biomedical Optics, 13, 4, 1-19

11. Jankowska M.A., Sypniewska-Kaminska G., 2012, Interval finite difference method for bioheat transfer problem given by the Pennes equation with uncertain parameters, Mechanics and Control, 31, 2, 77-84

12. JASIŃSKI M., 2014, Modelling of tissue thermal injury formation process with application of direct sensitivity method, Journal of Theoretical and Applied Mechanics, 52, 947-957

13. JAsińsKi M., 2015, Modelling of thermal damage in laser irradiated tissue, Journal of Applied Mathematics and Computational Mechanics, 14, 67-78

14. JAsiński M., 2018, Numerical analysis of soft tissue damage process caused by laser action, AIP Conference Proceedings, 060002, 1922

15. Jasiński M., Majchrzak E., Turchan L., 2016, Numerical analysis of the interactions between laser and soft tissues using generalized dual-phase lag model, Applied Mathematic Modelling, 40, $2,750-762$

16. KaŁuża G., Majchrzak E., Turchan L., 2017, Sensitivity analysis of temperature field in the heated soft tissue with respect to the perturbations of porosity, Applied Mathematical Modelling, 49, 498-513 
17. Di Lizia P., Armellin R., Bernelli-Zazzera F., Berz M., 2014, High order optimal control of space trajectories with uncertain boundary conditions, Acta Astronautica, 93, 217-229

18. Majchrzak E., Mochnacki B., 2016, Dual-phase lag equation. Stability conditions of a numerical algorithm based on the explicit scheme of the finite difference method, Journal of Applied Mathematics and Computational Mechanics, 15, 89-96

19. Majchrzak E., Mochnacki B., 2017, Implicit scheme of the finite difference method for 1D dual-phase lag equation, Journal of Applied Mathematics and Computational Mechanics, 16, 3, $37-46$

20. Majchrzak E., Turchan L., Dziatkiewicz J., 2015, Modeling of skin tissue heating using the generalized dual-phase lag equation, Archives of Mechanics, 67, 6, 417-437

21. MARKov S.M., 1995, On directed interval arithmetic and its applications, Journal of Universal Computer Science, 1, 514-526

22. Mochnacki B., Ciesielski M., 2016, Sensitivity of transient temperature field in domain of forearm insulated by protective clothing with respect to perturbations of external boundary heat flux, Bulletin of the Polish Academy of Sciences - Technical Sciences, 64, 3

23. Mochnacki B., PiaseckA-Belkhayat A., 2013, Numerical modeling of skin tissue heating using the interval finite difference method, Molecular and Cellular Biomechanics, 10, 3, 233-244

24. Mochnacki B., Suchy J.S., 1995, Numerical Methods in Computations of Foundry Processes, PFTA, Cracow

25. Moore R.E., 1966, Interval Analysis, Prentice-Hall, Englewood Cliffs

26. NAKAo M., 2017, On the initial-boundary value problem for some quasilinear parabolic equations of divergence form, Journal of Differential Equations, 263, 8565-8580

27. PARuch M., 2014, Hyperthermia process control induced by the electric field in order to destroy cancer, Acta of Bioengineering and Biomechanics, 16, 4, 123-130

28. Piasecka-Belkhayat A., 2011, Interval Boundary Element Method for Imprecisely Defined Unsteady Heat Transfer Problems (in Polish), Silesian University of Technology, Gliwice

29. Piasecka-Belkhayat A., Jasiński M., 2011, Modelling of UV laser irradiation of anterior part of human eye with interval optic, [In:] Evolutionary and Deterministic Methods for Design, Optimization and Control. Applications, Burczyński T., Périaux J. (Eds.), CIMNE, Barcelona, 316-321

30. Piasecka-Belkhayat A., Korczak A., 2016, Numerical modelling of the transient heat transport in a thin gold film using the fuzzy lattice Boltzmann method with alpha-cuts, Journal of Applied Mathematics and Computational Mechanics, 15, 1, 123-135

31. Piasecka-Belkhayat A., Korczak A., 2017, Modeling of thermal processes proceeding in a 1D domain of crystalline solids using the lattice Boltzmann method with an interval source function, Journal of Theoretical and Applied Mechanics, 55, 1, 167-175

32. Popova E.D., 1994, Extended interval arithmetic in IEEE floating-point environment, Interval Computations, 4, 100-129

33. Popova E.D., 2011, Multiplication distributivity of proper and improper intervals, Reliable Computing, 7, 129-140

34. Welch A.J., 2011, Optical-Thermal Response of Laser Irradiated Tissue, M.J.C. van Gemert (Eds.), 2nd edit., Springer 